\title{
Integration of Fractal Biosensor in a Digital Microfluidic Platform
}

\author{
Yousof Mashraei, Shilpa Sivashankar, Ulrich Buttner, Khaled Nabil Salama \\ Computer, Electrical And Mathematical Sciences and Engineering (CEMSE) \\ King Abdullah University of Science and Technology (KAUST) \\ Thwual 23955-6900, Kingdom of Saudi Arabia \\ yousof.mashraei@kaust.edu.sa
}

\begin{abstract}
Fractal capacitive electrodes have been successfully integrated into a digital microfluidic open-platform. These electrodes perform actuation and withstand voltages up to $300 \mathrm{~V}$ without insulation-layer breakdown. They were used to quantify the concentration levels of $C$-reactive protein (CRP) to determine the risk of cardiovascular disease. The capacitance increased sevenfold and stabilized in less than 5 minutes. The sensor shows a decreasing trend of capacitance readouts with the increase of concentrations. The same immunoassay was tested with untreated electrodes and showed no significant response, which suggests that immobilization was necessary. This configuration allows the electrodes to be used as biosensors.
\end{abstract}

Keywords-Fractal Capacitor; Biosensor; Digital Microfluidic; EWOD; Electrowetting On Dielectric; C-Reactive Protein; CRP

\section{INTRODUCTION}

Medical-care services are becoming less expensive with advances in biomedical technology. Yet, this improvement falls beyond the reach of poor countries that have low education standards and, accordingly, low health awareness [1]. Hence, a reliable and low-cost medical-diagnostic platform would meet an important need. The C-reactive-protein (CRP) test is a measure of vascular inflammation and can, therefore, be used to detect cardiovascular diseases [2]. CRP can be quantified with a diagnostic blood-screening test. It has generated considerable attention, as it can provide a good indication of many health conditions, including heart diseases [3, 4]. Though traditional assays are still dominant, the demand is increasing for miniaturized and affordable assay systems. Such systems must have fast-response and relatively low-cost operations with an easy-to-use interface. These requirements can be met in a digital microfluidic platform, which is a type of lab-on-chip (LOC) system. It is a system that utilizes the electrowetting-ondielectric (EWOD) phenomenon and works at the microliter to picoliter scale. Thus, the analyte dosage can be substantially reduced. The system also introduces several other advantages and functionalities into the bioassays [5]. So far, sensors have not integrated efficiently with these systems. Moreover, the integrated sensors are usually bulky and require sophisticated instrumentation. This makes the system economically unfeasible, especially for third-world countries [6]. On the other hand, capacitive sensors are becoming more popular in bioassays because of their simple structure, their capability for integration, and their reliable sensitivity and flexibility.
As an established technology, it provides many analysis choices with high accuracy and practical measurement process. As a result, these capacitive sensors have been implemented in many applications, including biomedical detection and have shown good results [7-10]. One type of capacitive sensor is the fractalshaped capacitor, which is reported to have less residual stress and provide a uniform distribution of the electrical field [11-13]. This can play a major role in EWOD systems. Consequently, digital microfluidic platforms approach commercial availability in biomedical applications by integrating consistent and inexpensive sensors.

In this work, fractal capacitive electrodes (FraC) were investigated as potential sensors to be integrated into digital microfluidic platforms. These FraCs can be used as actuation electrodes and provide the "sense-while-actuate" ability to the system. In this way, the usage flexibility of the electrodes can be enhanced significantly while lowering the total fabrication cost. Also, the effect of the electrical field required for the actuation can be identified and examined for the sample under test. Finally, the FraC can be used to send feedback signals to the control unit with droplet information (e.g., concerning droplet size and location), which can improve the control efficiency over analyte doses.

\section{EXPERIMENTAL PROCEDURE}

\section{A. Digital Microfluidic Fabrication}

As illustrated in Fig. 1, the digital microfluidic platform was fabricated in KAUST NanoFab using a standard in-house photolithography procedure. The N-type wafer was firstly precleaned in piranha solution $\left(\mathrm{H}_{2} \mathrm{SO}_{4}: \mathrm{H}_{2} \mathrm{O}_{2} 4: 1\right)$ for 10 minutes and dried in a nitrogen drier for 5 minutes. Then it was put in a Tystar ${ }^{\circledR} 3$ Tube Furnace Bank system to thermally grow the isolation layer of $2 \mu \mathrm{m}$-thick silicon dioxide $\left(\mathrm{SiO}_{2}\right)$. After deposition of the isolation layer, the wafer was cleaned and set in an ESC ${ }^{\circledR}$ Reactive \& Metal Sputter System chamber to deposit the metal layer using the DC sputter deposition technique. The metal layer consists of an approximately $15 \mathrm{~nm}$ thick layer of titanium (Ti) that works as an adhesive promoter, and an approximately $150 \mathrm{~nm}$-thick layer of platinum $(\mathrm{Pt})$ that works as the active metal layer. After the metal layer was deposited, the wafer was spin-coated with the standard AZ1512 photoresist to attain a $1.4 \mu \mathrm{m}$-thick layer for the photolithography process. The wafer was then exposed to UV source in the 
EVG $6200 \infty$ Mask Aligner® system to pattern the photoresist. Next, the photoresist was developed in AZ 726 MIF developer to expose the metal layer underneath. The wafer was then placed in an Oxford ${ }^{\circledR}$ Instruments PlasmaLab System100 Plasma Etcher to completely etch the exposed metal layer. The Acetone and IPA agitation technique was used to remove most of the photoresist layer on the wafer. Next, a descum etching process was used to ensure the removal of all the residues of the photoresist. Using Oxford ${ }^{\circledR}$ Instruments FlexAL system, the insulation layer was deposited by the Atomic Layer Deposition (ALD) technique. A $100 \mathrm{~nm}$ hafnium dioxide $\left(\mathrm{HfO}_{2}\right)$ dielectric layer was deposited at $300^{\circ} \mathrm{C}$ and $80 \mathrm{mTorr}$ for 5000 cycles. Then, to achieve the hydrophobic surface for EWOD phenomenon, a $\sim 500 \mathrm{~nm}$ thick layer of Teflon was deposited by casting the Teflon, spin coating it at 2000rpm, then baking it at $165^{\circ} \mathrm{C}$ for 30 minutes.

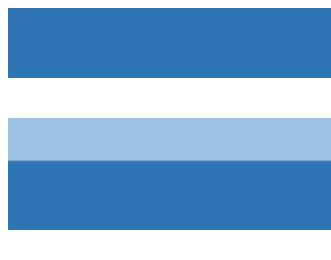

a) Silicon wafer

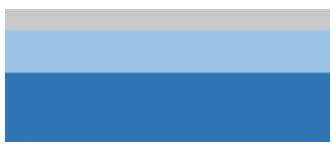

c) Metal-layer deposition by PVD sputtering (Ti:15nm)/(Pt:150nm)

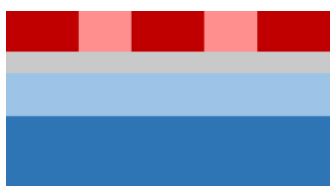

d) Photoresist coating and patterning by UV exposure

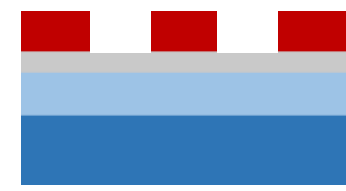

e) Develop the photoresist and expose the metal layer underneath

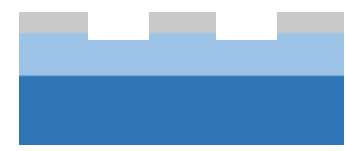

f) Dry etch the exposed metal layer and strip off the photoresist

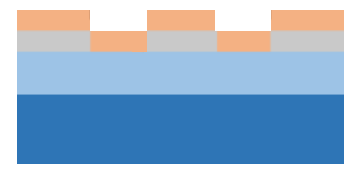

g) Dielectric layer deposition by ALD ( $\left.\mathrm{HfO}_{2}: 100 \mathrm{~nm}\right)$

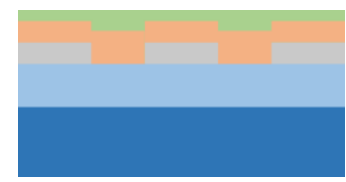

g) Spin coating of Teflon layer $(0.5 \mu \mathrm{m})$

Fig. 1. Fabrication Process of the Digital Microfluidic Platform.

\section{B. Measurement Setup}

The measurement setup consists of a computer that controls the other instruments and acquires measurement data for analysis. The high-voltage source used for the actuation tests is the LabSmith ${ }$ HVS448 High Voltage Sequencer. It can reach a voltage of up to $3000 \mathrm{~V}$ and provide feedback measurement of the voltage and current on the sample. The LCR meter used to measure the change in capacitance is the Agilent ${ }^{\circledR}$ E4980A Precision LCR meter, which is used to measure the capacitance of the bioassay described later, see Fig. 2.

\section{Immobilization of Anti-CRP Antibodies}

The biosensors require immobilization of CRP antibodies to the surface to quantify CRP. Only the biosensors surface was selectively treated for the immobilization, whereas the other electrodes were left untreated; to carry out the actuating function effectively. The immobilization of antibodies is a major step for the CPR quantification test. This process consists of three major steps, illustrated in Fig. 3. First, during Teflon coating, selected

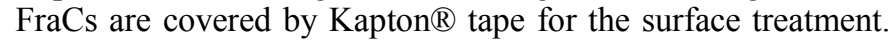
These FraCs are cleaned and then immersed in an ethanol solution of $10 \mathrm{mM}$ mercatopropionic acid (MPA) for $24 \mathrm{hrs}$. Then they are washed with distilled water and allowed to dry. Second, they are treated for $4 \mathrm{hrs}$ with a solution that contains $0.05 \mathrm{M}$ EDC and $0.03 \mathrm{M}$ NHS to form self-assembled monolayers (SAM). Next, a $2 \mu 1$ drop of CRP antibodies is added and allowed to settle on the surface for $1 \mathrm{hr}$. Then it is washed with distilled water to remove any unattached antibodies.
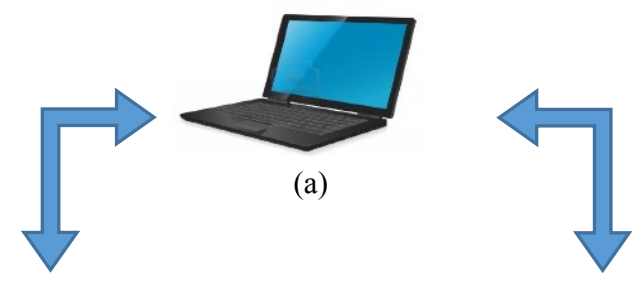

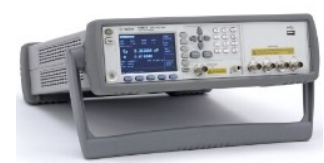

(d)

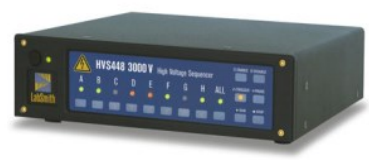

(b)

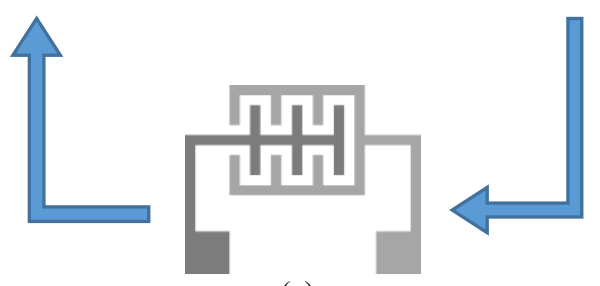

(c)

Fig. 2. The measurement setup which consists of (a) control unit, (b) highvoltage source (LabSmith ${ }^{\circledR}$ HVS448 High Voltage Sequencer), (c) the tested digital microfluidic chip, and (d) LCR meter (Agilent ${ }^{\circledR}$ E4980A Precision LCR Meter). 
(a) FraC surface without

Teflon layer

(b) Coated with $10 \mathrm{mM}$ MPA for $24 \mathrm{hrs}$

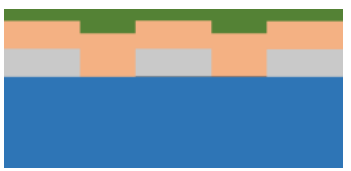

(c) Incubate with EDC/NHS for $4 \mathrm{hrs}$

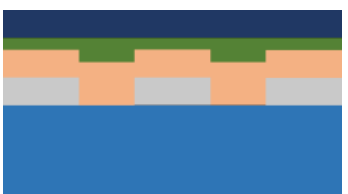

(d) Coat anti-CRP

antibodies

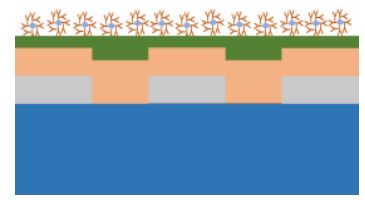

(e) Immobilize of anti-CRP antibodies on SAM layer

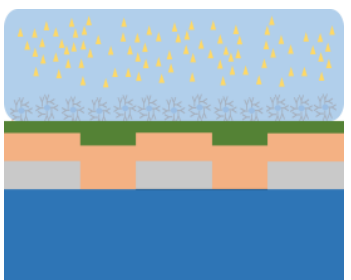

(f) Add the human CRP

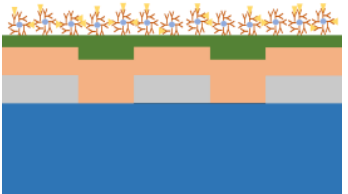

(g) Attachment of the CRP to the antibodies

Fig. 3. (a-e) Surface treatment procedure to prepare the biosensor, $(f, g)$ Antigen-antibody interactions.

\section{RESULTS AND DISCUSSION}

\section{A. Actuation Experiment}

This experiment was conducted to evaluate the reliability and endurance of the $\mathrm{FraC}$ when subjected to high voltages. Two FraC designs were tested in this experiment: FraC3, which has an area of $1.1 \times 1.1 \mathrm{~mm}^{2}$ and $3 \mu \mathrm{m}$ spacing; and FraC6, which has an area of $2.2 \times 2.2 \mathrm{~mm}^{2}$ and $6 \mu \mathrm{m}$ spacing. The actuation test was carried out by adding a $4 \mu \mathrm{l}$ water droplet on the FraC6 and applying a high voltage to the microfluidic device. The actuation of the droplet was successful at $100 \mathrm{~V}$ and $2 \mathrm{~Hz}$ frequency, see Fig. 4. The insulation layer of $\mathrm{HfO}_{2}$ was further tested by increasing the voltage on the FraC terminals while evaluating the droplet behavior. The actuation was sustainable for voltages that reached the $300 \mathrm{~V}$ level without a significant sign of insulation-layer breakdown.
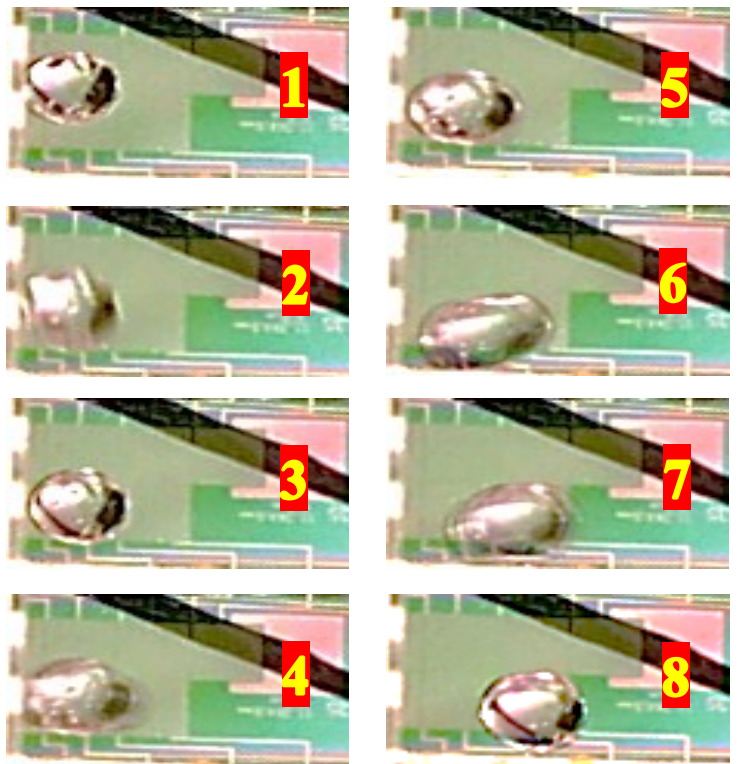

Fig. 4. Image sequence of a movie that shows the actuation performed by the FraC6 with $100 \mathrm{~V}$ and $2 \mathrm{~Hz}$.

\section{B. CRP Quantification Experiment}

In this experiment, two sensors were employed to quantify the concentrations: untreated FraCs, and surface-treated FraCs "biosensors". First, the agglutination progress with time was observed and used to estimate the duration of the measurements, see Fig. 5. After the immobilization of anti-CRP antibodies on FraC3 devices, a commercial CRP solution was diluted to obtain different concentrations $(5,7.5,10) \mathrm{mg} / \mathrm{L}$. Then a $2 \mu \mathrm{l}$ drop of each concentration was added onto different sensors, and the capacitance measurement was acquired through the control unit. The capacitance was measured at $1 \mathrm{kHz}$ for approximately 15 minutes.

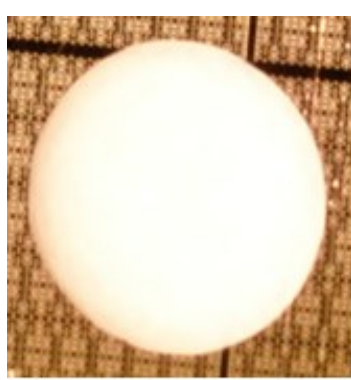

(a)

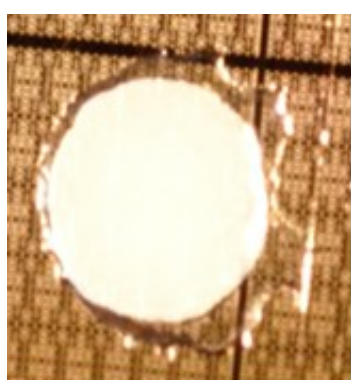

(c)

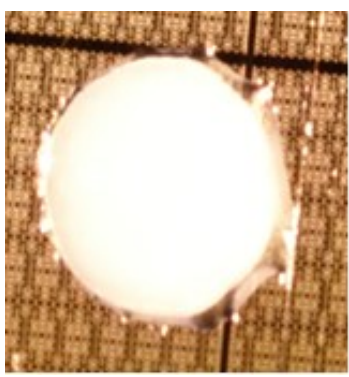

(b)

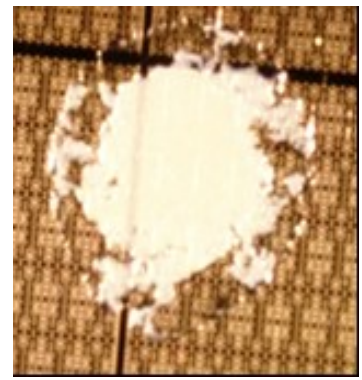

(d)
Fig. 5. Agglutination progress with time:(a) 0 minute, (b) 2 minutes, (c) 9 minutes, (d) 25 minutes. 


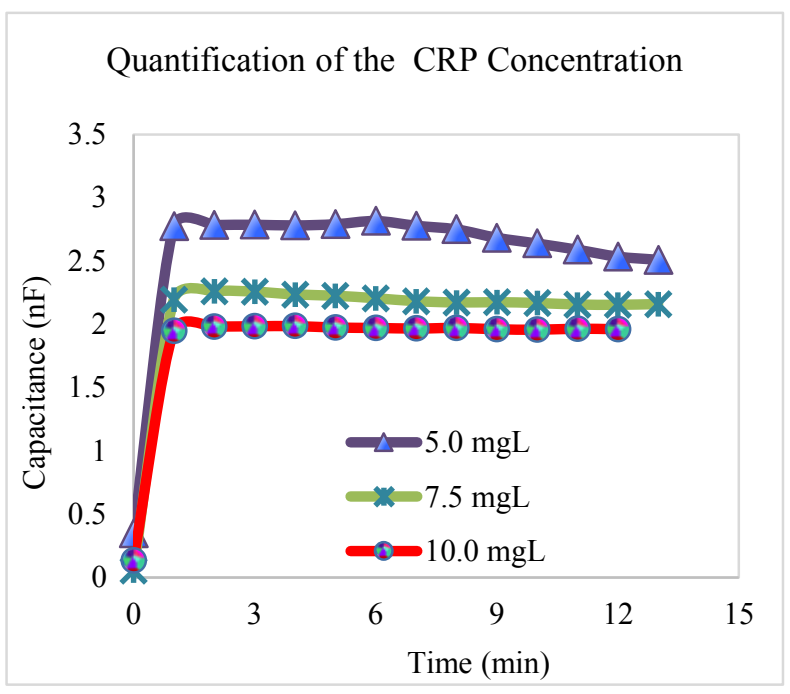

Fig. 6. Capacitance readout measurements of CRP concentration levels.

The biosensor measurements showed a fast response in capacitance of over a sevenfold increase, which indicates high sensitivity to CRP concentrations. However, the capacitance level has an inverse relationship to the concentrations, as plotted in Fig. 6. This can be interpreted as a dielectric reduction due to more antigen-antibody interactions. The capacitance tends to decrease with time as a result of the serum drying out. Untreated FraC measurements showed no significant sensitivity to the CRP concentrations as the capacitance was slightly dropped. This result suggests that the antibody-immobilization step is crucial to the CRP quantification.

\section{CONCLUSION}

Fractal capacitive electrodes were successfully fabricated and integrated into a digital microfluidic open platform. The FraCs were functional in performing droplet actuation with high applied voltages. The actuation experiment shows water droplet actuation starting at $100 \mathrm{~V}$ and a frequency of $2 \mathrm{~Hz}$. The same test indicates a good dielectric property of the insulation layer. The FraCs were used as a biosensor by applying a surface treatment to selected electrodes. They were used to detect the CRPconcentration levels in a controlled manner. The CRP quantification experiment shows a fast response of the biosensor with high sensitivity to the CRP. It also shows a decreasing trend of the capacitance readouts as the CRP-concentration level increases in the sample. However, the untreated-FraCs measurement shows no significant response with a minor drop of the capacitance readout. This confirms the importance of the surface-treatment step for enhancing the sensitivity of the capacitive sensor.

\section{REFERENCES}

[1] I. N. Okeke, "Diagnostic insufficiency in Africa," Clinical infectious diseases, vol. 42, pp. 1501-1503, 2006.

[2] K. Sato, M. Tokeshi, H. Kimura, and T. Kitamori, "Determination of carcinoembryonic antigen in human sera by integrated bead-bed immunoasay in a microchip for cancer diagnosis," Analytical Chemistry, vol. 73, pp. 1213-1218, 2001.

[3] W.-B. Lee, Y.-H. Chen, H.-I. Lin, S.-C. Shiesh, and G.-B. Lee, "An integrated microfluidic system for fast, automatic detection of C-reactive protein," Sensors and Actuators B: Chemical, vol. 157, pp. 710-721, 2011.

[4] Y.-N. Yang, H.-I. Lin, J.-H. Wang, S.-C. Shiesh, and G.-B. Lee, "An integrated microfluidic system for C-reactive protein measurement," Biosensors and Bioelectronics, vol. 24, pp. 3091-3096, 2009.

[5] M. Abdelgawad and A. R. Wheeler, "The digital revolution: a new paradigm for microfluidics," Advanced Materials, vol. 21, pp. 920-925, 2009.

[6] L. Kin Fong, "Microfluidics in Detection Science: Lab-on-a-chip Technologies," Microfluidics in Detection Science: Lab-on-a-chip Technologies, pp. 1-28, 2015.

[7] A. Qureshi, Y. Gurbuz, and J. H. Niazi, "Capacitive aptamer-antibody based sandwich assay for the detection of VEGF cancer biomarker in serum," Sensors and Actuators B: Chemical, vol. 209, pp. 645-651, 2015.

[8] C. Sapsanis, S. Sivashankar, H. Omran, U. Buttner, and K. N. Salama, "Capacitive immunosensor for C-reactive protein quantification," IEEE InternationalMidwest Symposium on Circuits and Systems (MWSCAS), Colorado, USA, 2015.

[9] X. Luo and J. J. Davis, "Electrical biosensors and the label free detection of protein disease biomarkers," Chemical Society Reviews, vol. 42, pp. 5944-5962, 2013.

[10] C. Sapsanis, H. Omran, V. Chernikova, O. Shekhah, Y. Belmabkhout, U. Buttner, et al., "Insights on Capacitive Interdigitated Electrodes Coated with MOF Thin Films: Humidity and VOCs Sensing as a Case Study," Sensors, vol. 15, pp. 18153-18166, 2015.

[11] A. M. Elshurafa, A. G. Radwan, A. Emira, and K. N. Salama, "RF MEMS fractal capacitors with high self-resonant frequencies," Microelectromechanical Systems, Journal of, vol. 21, pp. 10-12, 2012.

[12] A. M. Elshurafa and K. N. Salama, "Two-layer RF MEMS fractal capacitors in PolyMUMPS for S-band applications." IET Micro \& Nano letters, vol 7, no 5, pp. 419-421, 2012.

[13] A. M. Elshurafa, P. Ho and K. N. Salama, "Modeling and fabrication of an RF MEMS variable capacitor with a fractal geometry," IEEE International Symposium on Circuits and Systems (ISCAS), pp 2711$2714,2013$. 